\title{
Water Quality Assessment of Paper Mills Effluent Discharge Areas
}

\author{
Md. Shakilur Zaman Shakil and M. G. Mostafa \\ Institute of Environmental Science, University of Rajshahi, Rajshahi 6205, Bangladesh
}

\begin{tabular}{|c|c|}
\hline Article's Information & Abstract \\
\hline $\begin{array}{l}\text { Received: } \\
\text { 12.08.2021 } \\
\text { Accepted: } \\
\text { 12.09.2021 } \\
\text { Published: } \\
\text { 30.09.2021 }\end{array}$ & $\begin{array}{l}\text { The study attempted to assess the water quality around paper mill effluents } \\
\text { discharge areas. Several physicochemical parameters and the Canadian Council } \\
\text { of Ministers of the Environment (CCME) Water Quality Index (WQI) were } \\
\text { considered to determine the pollution level of surface and groundwater in the } \\
\text { selected paper mills areas located in Saidpur, Gobindaganj, and Dupchanchia } \\
\text { Upazilas of Bangladesh. Physicochemical characterization of the surface water } \\
\text { around the paper mills areas showed that the concentration of EC, TSS, BOD, }\end{array}$ \\
\hline $\begin{array}{l}\text { Keywords: } \\
\text { Paper mills } \\
\text { Effluent } \\
\text { CCME WQI } \\
\text { Impacts } \\
\text { Bioaccumulation }\end{array}$ & 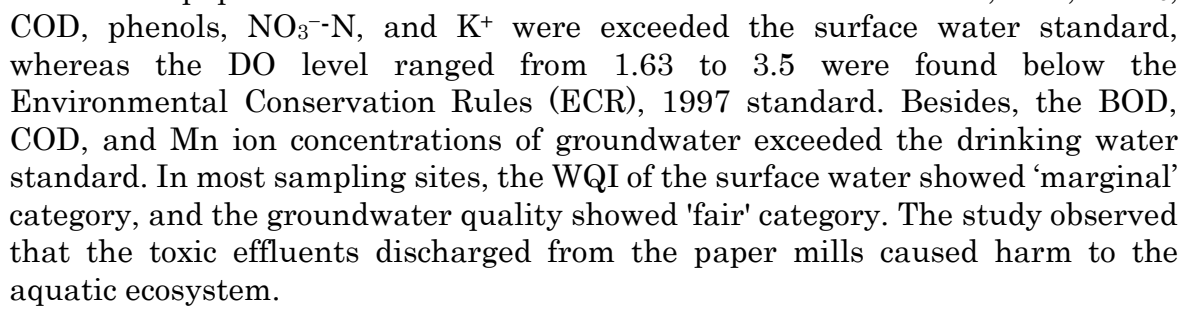 \\
\hline
\end{tabular}

DOI: 10.22401/ANJS.24.3.10

*Corresponding author: mgmostafa@ru.ac.bd

\section{Introduction}

Paper production is increasing rapidly all over the world. The world average per capita paper consumption increased from $49 \mathrm{~kg}$ in 1995 to $53 \mathrm{~kg}$ in 2018 [1-3]. There is a strong positive correlation was observed between the paper consumption rate and the gross national product (GNP). The per capita paper and board consumption rate is $5 \mathrm{~kg}$ in Bangladesh whereas, this rate is more than $200 \mathrm{~kg}$ in advanced countries, and the world average rate is about 53 $\mathrm{kg}$ in 2018 [2]. The average paper consumption rate is increasing in developing countries as well as Bangladesh. The pulp and paper sector were dependent upon BCIC (Bangladesh Chemical Industry Corporation). There was four government-owned paper mill in Bangladesh named Karnaphuli Paper Mill (KPM) at Chandragona in Chittagong, Khulna Newsprint Mill (KNM) at Khulna, North Bengal Paper Mill (NBPM) at Pakshi and Sylhet Pulp and Paper Mill (SPPM) at Sylhet. Among them, only KPM is in operation and producing nearly 30,000 tonnes of paper annually. Today KPM is producing less than $5 \%$ of the total paper used in Bangladesh. The private entrepreneur has taken over the paper-producing sector in the country. Due to the lack of raw materials paper mills in Bangladesh use imported pulp and recycled fiber for paper production [4]. The number of paper mills in Bangladesh is 110, and the annual production capacity of these mills is more than 15 lakh tons. The country's internal demand is 9-10 lakh tons.
Therefore, a decade ago paper mills have started exporting their products [5].

Paper mills are one of the most effluent discharging industries in the world. Paper mills consume a huge volume of freshwater [6]. Its water consumption rate is $150-250 \mathrm{~m}^{3} /$ ton of paper production $[7,8]$. Its effluent discharge rate is $150-200 \mathrm{~m}^{3} /$ ton of paper production $[9,10]$. Effluent management in mills and factories is a global concern. Maximum industries discharge their untreated or poorly treated effluent into nearby surface water body which causes water quality deterioration [11-12]. The fundamental steps of the paper manufacturing process are raw material preparation, digestion, pulping, and bleaching. The raw materials of paper are cellulose, wood, or recycled paper. Cellulosic fibers originated from non-wood raw materials such cereal straw, esparto grass, reeds, jute, and sisal are the other sources of pulping raw materials [13]. In a paper mill, the raw materials are mixed with water to produce fiber suspension. Pulping is a vital part of paper production. Pulp and paper quality depend upon the pulping process. Various types of pulping processes are used to get a variety of quality of the paper. The pulping processes are mainly divided into four types: mechanical, chemical, recycled fiber, and textile fiber pulp. These types can further be classified into subgroups. Every group and sub-group have a specific use of chemicals. A lot of chemicals are used in these pulping processes. Generally, almost 200 chemicals are used effectively are used as additives, fillers, 


\section{Al-Nahrain Journal of Science}

ANJS, Vol.24 (3), September, 2021, pp. 63-72

strengtheners, whiteners, dyes, surfactants, biocides in the paper production process [14]. The raw material preparation, pulping, washing, screening, bleaching, and coating are the main environmental pollution sources of the pulp and paper mills. All the production stage wastewater has toxic components with different characterization. As a result, the paper mill effluent contains a high level of EC, COD, BOD, TOC, TDS, TSS, inorganic ions, heavy metals, organic compounds, etc. [15-16].

The water quality index (WQI) is a good communication tool to transfer water quality data [17]. Several water quality indices have been developed to assess water quality. The Canadian Council of Ministers of the Environment (CCME) developed a water quality index to summarize water quality data. Considering the water quality data, the CCME WQI gives a result in a single number between zero to a hundred [18]. The objective of this work was to assess the water quality around the paper mills' effluents discharge areas.

\section{Methodology}

\subsection{Study area}

The study was conducted at three paper mill areas located in Saidpur (PM-1), Gobindaganj (PM-2), and Dupchanchia
(PM-3) Upazilas of Rangpur, Gaibandha, and Bogura Districts of Bangladesh. These paper mills were established very recently, used waste papers and imported pulps as raw materials. PM-1, PM-2, and PM-3 discharge effluents into the river Kharkharia, canal Isamoti Gojari, and river Nagar, respectively.

\subsection{Sample collection}

Surface water samples were collected from the paper mills effluent discharge point (S-1), 350-meter downstream (S-2), and 700-meter downstream (S-3) of the three (3) paper mills areas. Groundwater samples were also collected from effluent discharge areas of the three (3) paper mills. The samples were collected three times (Pre-monsoon, Monsoon, and Post-monsoon) in a year from the monsoon2019 to premonsoon-2021. The total number of surface and ground water samples was 54 and 18, respectively. The samples were collected in clean plastic bottles and stored in a refrigerator to avoid degradation before the chemical analysis.

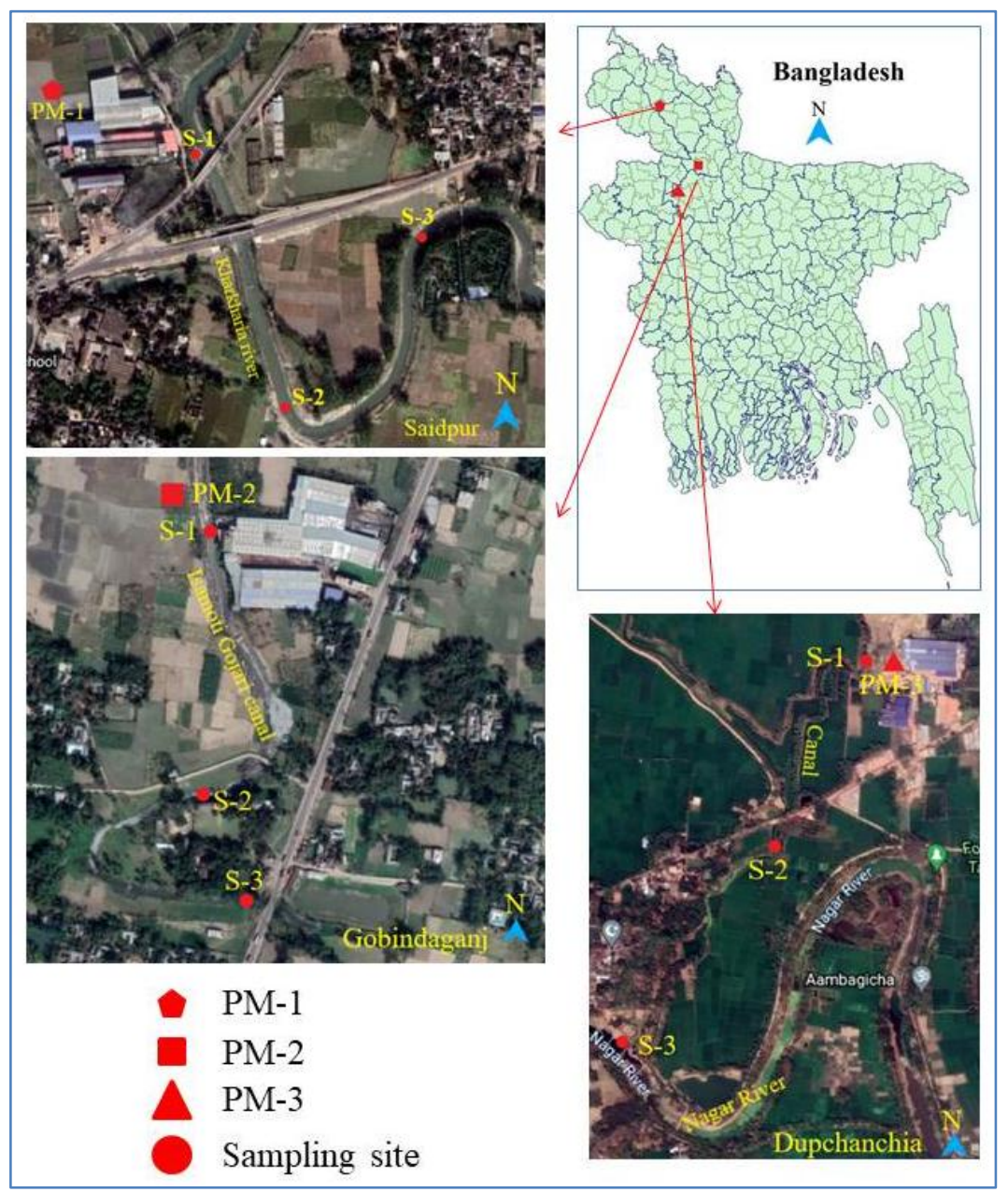

Figure 1. Study area (Source: Google earth). 


\section{Al-Nahrain Journal of Science}

ANJS, Vol.24 (3), September, 2021, pp. 63-72

\subsection{Physicochemical analysis}

The study considered a total of 27 physicochemical parameters i.e., Temperature, $\mathrm{pH}, \mathrm{DO}, \mathrm{EC}$, turbidity, TSS, TDS, BOD 5 , COD, TOC, Phenols, $\mathrm{Cl}^{-}, \mathrm{SO}_{4}{ }^{2-}, \mathrm{NO}_{3}{ }^{-}-\mathrm{N}$, $\mathrm{PO}_{4}{ }^{3-}, \mathrm{HCO}_{3}{ }^{-}, \mathrm{Na}^{+}, \mathrm{K}^{+}, \mathrm{Ca}^{2+}, \mathrm{Mg}^{2+}, \mathrm{Fe}, \mathrm{Cu}, \mathrm{Zn}, \mathrm{Mn}, \mathrm{Pb}, \mathrm{Cr}$, and $\mathrm{Cd}$. Among the parameters, temperature, $\mathrm{pH}, \mathrm{DO}, \mathrm{EC}$, and turbidity were measured directly by digital multi-meters in the field. $\mathrm{SO}_{4}{ }^{2-}, \mathrm{NO}_{3}{ }^{-}-\mathrm{N}, \mathrm{PO}_{4}{ }^{3-}$ and Phenols were measured using the UV-spectrophotometric method. $\mathrm{Na}^{+}$, $\mathrm{K}^{+}, \mathrm{Ca}^{2+}, \mathrm{Mg}^{2+}, \mathrm{Fe}, \mathrm{Cu}, \mathrm{Zn}, \mathrm{Mn}, \mathrm{Pb}, \mathrm{Cr}$, and $\mathrm{Cd}$ were measured using the atomic absorption spectrophotometer model: Shimadzu, AA-7000. Other parameters were measured using the standard chemical methods [19].

\subsection{Water Quality Index (WQI)}

The Canadian Council of Ministers of the Environment (CCME) Water Quality Index (WQI) was used to assess the water quality in the study area. A total of twenty two (22) water quality parameters (Temperature, $\mathrm{pH}, \mathrm{DO}, \mathrm{EC}, \mathrm{TSS}$, TDS, BOD 5 , COD, Phenols, $\mathrm{Cl}^{-}, \mathrm{SO}_{4}{ }^{2-}, \mathrm{NO}_{3}{ }^{-}-\mathrm{N}, \mathrm{Na}^{+}, \mathrm{K}^{+}$, $\mathrm{Ca}^{2+}, \mathrm{Fe}, \mathrm{Cu}, \mathrm{Zn}, \mathrm{Mn}, \mathrm{Pb}, \mathrm{Cr}$, and $\mathrm{Cd}$ ) were considered to calculate the WQI score of surface water because turbidity, TOC, $\mathrm{PO}_{4}{ }^{3-}, \mathrm{HCO}_{3}{ }^{-}$, and $\mathrm{Mg}^{2+}$ have no surface water standard in Bangladesh. A total of 24 parameters (Temperature, $\mathrm{pH}, \mathrm{DO}$, turbidity, TSS, TDS, BOD 5 , COD, Phenols, $\mathrm{Cl}^{-}, \mathrm{SO}_{4}{ }^{2-}, \mathrm{NO}_{3}{ }^{-}-\mathrm{N}, \mathrm{PO}_{4}{ }^{3-}, \mathrm{Na}^{+}, \mathrm{K}^{+}, \mathrm{Ca}^{2+}, \mathrm{Mg}^{2+}$, $\mathrm{Fe}, \mathrm{Cu}, \mathrm{Zn}, \mathrm{Mn}, \mathrm{Pb}, \mathrm{Cr}$, and $\mathrm{Cd}$ ) were considered to calculate the WQI score of groundwater because EC, TOC, and $\mathrm{HCO}_{3}{ }^{-}$have no drinking water standard in Bangladesh.

The CCME WQI comprises three factors to calculate i.e., Scope $\left(F_{1}\right)$, frequency $\left(F_{2}\right)$, and amplitude $\left(F_{3}\right)[18,20$, 21]. $F_{1}$ represents the percentage of parameters that do not meet their objectives at least once (failed parameters) during the period (Equation i). $F_{2}$ represents the percentage of individual tests that do not meet their objectives (failed tests) (Equation ii) and $\mathrm{F}_{3}$ represents the amount by which failed tests do not meet their objectives (Equation vi). The equations are given bellow:

$$
\begin{aligned}
& \text { Scope, } \mathrm{F}_{1}=\left(\frac{\text { Number of Failed Variables }}{\text { Total Number of Variables }}\right) \times 10 \\
& \text { Frequency, } \mathrm{F}_{2}=\left(\frac{\text { Number of Failed Tests }}{\text { Total Number of Tests }}\right) \times 100
\end{aligned}
$$

Amplitude, $\mathrm{F}_{3}$ : Amplitude is calculated based on the excursion of each failed test related to its objective.

If failed test greater than objective:

Excursion $=\left(\frac{\text { Failed Test value }}{\text { Objective }}\right)-1$

If failed test less than objective:

Excursion $=\left(\frac{\text { Objective }}{\text { Failed Test value }}\right)-1$

Normalized Sum of Excursions (NSE) = $\left(\frac{\sum \text { Excursion }}{\text { Total Number of Test }}\right)$

Amplitude, $\mathrm{F}_{3}=\left(\frac{\mathrm{NSE}}{0.01 \mathrm{NSE}+0.01}\right)$

CCME WQI calculation:

$$
\text { CCME WQI }=100-\left(\frac{\sqrt{\mathrm{F}^{2}+\mathrm{F}^{2}+\mathrm{F}^{2}}}{1.732}\right)
$$

Then the calculated WQI score was ranked into one of the following categories, i.e., Excellent (95-100), good (80-94), fair (65-79), marginal (45-64), and poor (0-44).

\section{Results and Discussion 3.1 Physicochemical characterization of surface water}

The physicochemical characteristics of surface water around the paper mill effluents discharge area are presented in Table 1,2, and 3. This study found that the temperature varied from 26.2 to $34.0{ }^{\circ} \mathrm{C}$, which were within the Bangladesh surface water standard (BD SWS). The mean $\mathrm{pH}$ values ranged from 7.18 to 7.85 within the permissible limit. The dissolved oxygen (DO) ranged from 1.63 to 3.5 $\mathrm{mg} / \mathrm{L}$ were below the required level. BOD load from paper mill effluents discharge caused DO depletion in surface water. A similar observation was reported by Mishra et al., 2013 [22].

The electrical conductivity (EC) values were 777.7 to $1853.3 \mu \mathrm{S} / \mathrm{cm}$ at different sites of the paper mill effluents discharge areas. At most of the sites, it exceeded the permissible limit. Several reports illustrated that the high EC value indicated the presence of various inorganic ions $[14,23]$. The turbidity ranged from 44.3 to 237.8 NTU. The total suspended solids (TSS) ranged from 168.6 to 564.5 $\mathrm{mg} / \mathrm{L}$ that exceeded the standard level. A similar study was reported by Mishra et al., 2013 [22].

The total dissolved solids (TDS) ranged from 511.3 to $1414.5 \mathrm{mg} / \mathrm{L}$ were found within the standard level. The biochemical oxygen demand $\left(\mathrm{BOD}_{5}\right)$ and chemical oxygen demand (COD) ranged from 136.2 to $238.5 \mathrm{mg} / \mathrm{L}$, and 274.8 to $699.3 \mathrm{mg} / \mathrm{L}$, respectively that exceeded the permissible limit. Organic and inorganic pollutants released from paper mill effluents resulted in a high volume of $\mathrm{BOD}_{5}$ and $\mathrm{COD}$ in the surface water. A similar study was reported by Giri et al. 2014 [16].

The total organic carbon (TOC) varied from 48 to 105 $\mathrm{mg} / \mathrm{L}$. Organic pollutants released from paper mill effluents resulted in high volume of TOC in the surface water. The phenolic compounds ranged from 1.145 to $2.302 \mathrm{mg} / \mathrm{L}$, which exceeded the standard level (Table 1). A similar report was observed by Toczyłowska-Mamińska, 2017 [23]. 


\section{Al-Nahrain Journal of Science}

ANJS, Vol.24 (3), September, 2021, pp. 63-72

\begin{tabular}{|c|c|c|c|c|c|}
\hline \multirow{2}{*}{ Parameter } & \multirow{2}{*}{$\begin{array}{l}\text { Sample } \\
\text { location }\end{array}$} & \multicolumn{3}{|c|}{ Mean \pm SD } & \multirow{2}{*}{ BD SWS [24] } \\
\hline & & PM-1 & PM-2 & PM-3 & \\
\hline \multirow{3}{*}{ Temperature } & S-1 & $34 \pm 2$ & $34.6 \pm 3.7$ & $34.7 \pm 3.3$ & \multirow{3}{*}{40} \\
\hline & $\mathrm{S}-2$ & $28.3 \pm 5.3$ & $29.2 \pm 5.2$ & $29 \pm 5.8$ & \\
\hline & $\mathrm{S}-3$ & $25.7 \pm 6.7$ & $26.8 \pm 7.2$ & $26.2 \pm 6.7$ & \\
\hline \multirow{3}{*}{$\mathrm{pH}$} & S-1 & $7.8 \pm 0.05$ & $7.85 \pm 0.05$ & $7.8 \pm 0.05$ & \multirow{3}{*}{$6.0-9.0$} \\
\hline & $\mathrm{S}-2$ & $7.4 \pm 0.1$ & $7.4 \pm 0.06$ & $7.5 \pm 0.07$ & \\
\hline & S-3 & $7.2 \pm 0.05$ & $7.18 \pm 0.04$ & $7.2 \pm 0.07$ & \\
\hline \multirow{3}{*}{ DO } & S-1 & $1.77 \pm 0.08$ & $1.63 \pm 0.15$ & $1.7 \pm 0.18$ & \multirow{3}{*}{$4.5-8.0$} \\
\hline & $\mathrm{S}-2$ & $2 \pm 0.13$ & $1.73 \pm 0.12$ & $1.9 \pm 0.25$ & \\
\hline & $\mathrm{S}-3$ & $3.5 \pm 1.1$ & $1.88 \pm 0.07$ & $3.5 \pm 0.81$ & \\
\hline \multirow{3}{*}{$\mathrm{EC}$} & S-1 & $1853.3 \pm 78.7$ & $1813.8 \pm 32.5$ & $1823.2 \pm 44.4$ & \multirow{3}{*}{1200} \\
\hline & $\mathrm{S}-2$ & $1364.8 \pm 77$ & $1595.7 \pm 56.4$ & $1397 \pm 77.5$ & \\
\hline & S-3 & $777.7 \pm 194.8$ & $1435.5 \pm 90.6$ & $836.8 \pm 154$ & \\
\hline \multirow{3}{*}{ Turbidity } & S-1 & $227.3 \pm 3.9$ & $233.8 \pm 10.8$ & $237.8 \pm 13.3$ & \multirow{3}{*}{-} \\
\hline & $\mathrm{S}-2$ & $138.8 \pm 31.2$ & $150 \pm 36.6$ & $159 \pm 35.6$ & \\
\hline & S-3 & $44.3 \pm 13.6$ & $56.6 \pm 20.4$ & $54.8 \pm 14.5$ & \\
\hline \multirow{3}{*}{ TSS } & S-1 & $551 \pm 19.5$ & $556.8 \pm 22.4$ & $564.5 \pm 7.9$ & \multirow{3}{*}{150} \\
\hline & $\mathrm{S}-2$ & $306 \pm 43.9$ & $464.2 \pm 33.4$ & $329.5 \pm 19.2$ & \\
\hline & $\mathrm{S}-3$ & $168.6 \pm 44.5$ & $383 \pm 31.3$ & $188.8 \pm 47.9$ & \\
\hline \multirow{3}{*}{ TDS } & S-1 & $1382 \pm 95.8$ & $1400 \pm 115$ & $1414.5 \pm 114$ & \multirow{3}{*}{2100} \\
\hline & $\mathrm{S}-2$ & $904 \pm 87.8$ & $955.2 \pm 80.6$ & $922.8 \pm 62.6$ & \\
\hline & $\mathrm{S}-3$ & $511.3 \pm 105.4$ & $585.7 \pm 161$ & $590.3 \pm 146.6$ & \\
\hline \multirow{3}{*}{$\mathrm{BOD}_{5}$} & S-1 & $227.2 \pm 21.5$ & $238.5 \pm 30.6$ & $233.6 \pm 29.7$ & \multirow{3}{*}{50} \\
\hline & $\mathrm{S}-2$ & $186.5 \pm 25.7$ & $219 \pm 27.9$ & $218.5 \pm 15.9$ & \\
\hline & $\mathrm{S}-3$ & $136.2 \pm 26.3$ & $195.7 \pm 24.7$ & $154.5 \pm 28.0$ & \\
\hline \multirow{3}{*}{ COD } & S-1 & $678.3 \pm 34$ & $699.3 \pm 52.3$ & $684.5 \pm 39.9$ & \multirow{3}{*}{200} \\
\hline & $\mathrm{S}-2$ & $464 \pm 110$ & $660.2 \pm 44.9$ & $528 \pm 130$ & \\
\hline & $S-3$ & $274.8 \pm 101$ & $606.7 \pm 58.2$ & $305.5 \pm 116.8$ & \\
\hline \multirow{3}{*}{ TOC } & S-1 & $99.5 \pm 3.4$ & $105 \pm 6.6$ & $105 \pm 7.8$ & \multirow{3}{*}{$10 *$} \\
\hline & $\mathrm{S}-2$ & $73.8 \pm 5.3$ & $84.6 \pm 11.1$ & $83.9 \pm 10.3$ & \\
\hline & S-3 & $48 \pm 2.4$ & $63.0 \pm 12.6$ & $50.7 \pm 3.7$ & \\
\hline \multirow{3}{*}{ Phenols } & S-1 & $2.302 \pm 0.79$ & $2.29 \pm 0.89$ & $2.17 \pm 0.59$ & \multirow{3}{*}{1} \\
\hline & $\mathrm{S}-2$ & $1.423 \pm 0.32$ & $1.816 \pm 0.55$ & $1.80 \pm 0.50$ & \\
\hline & S-3 & $1.145 \pm 0.24$ & $1.52 \pm 0.35$ & $1.36 \pm 0.39$ & \\
\hline
\end{tabular}

*[WHO standard $]$

The chloride $\left(\mathrm{Cl}^{-}\right)$and sulfate $\left(\mathrm{SO}_{4}{ }^{2-}\right)$ varied from 65.3 to $199.4 \mathrm{mg} / \mathrm{L}$ and 47 to $113.85 \mathrm{mg} / \mathrm{L}$, respectively were found within the permissible limit. The nitrate-nitrogen $\left(\mathrm{NO}_{3}{ }^{-}-\mathrm{N}\right)$ varied from 10.3 to $24.46 \mathrm{mg} / \mathrm{L}$, which exceeded the permissible limit at most of the sampling sites around the paper mill areas. A similar report was observed by Chandra et al., 2018 [25]. The phosphate $\left(\mathrm{PO}_{4}{ }^{3-}\right)$ and bicarbonate $\left(\mathrm{HCO}_{3}{ }^{-}\right)$varied from 1.885 to $7.08 \mathrm{mg} / \mathrm{L}$ and 30.07 to $95.44 \mathrm{mg} / \mathrm{L}$, respectively. But phosphate and bicarbonate have no surface water standard in Bangladesh. The sodium $\left(\mathrm{Na}^{+}\right)$and potassium $\left(\mathrm{K}^{+}\right)$ions ranged from 15.8 to $35.7 \mathrm{mg} / \mathrm{L}$, and 25.77 to $68.9 \mathrm{mg} / \mathrm{L}$, respectively. The sodium ion concentration was within the standard level, whereas, the potassium ion exceeded the permissible limit. A similar observation was made by Kumar et al., 2003 [26]. The calcium $\left(\mathrm{Ca}^{2+}\right)$ and magnesium $\left(\mathrm{Mg}^{2+}\right)$ ions concentration ranged from 10.29 to $29.3 \mathrm{mg} / \mathrm{L}$ and 2.73 to $7.81 \mathrm{mg} / \mathrm{L}$, respectively. The calcium ion was within the standard limit, whereas magnesium ion has no surface water standard in Bangladesh (Table 2). 


\section{Al-Nahrain Journal of Science}

ANJS, Vol.24 (3), September, 2021, pp. 63-72

\begin{tabular}{|c|c|c|c|c|c|}
\hline \multirow{2}{*}{ Parameter } & \multirow{2}{*}{$\begin{array}{l}\text { Sample } \\
\text { location }\end{array}$} & \multicolumn{3}{|c|}{ Mean \pm SD } & \multirow{2}{*}{ BD SWS [24] } \\
\hline & & PM-1 & PM-2 & PM-3 & \\
\hline \multirow{3}{*}{$\mathrm{Cl}^{-}$} & S-1 & $186.7 \pm 14.0$ & $197.1 \pm 17.3$ & $199.4 \pm 18.7$ & \multirow{3}{*}{600} \\
\hline & S-2 & $125.6 \pm 25.9$ & $138.4 \pm 32.5$ & $146.5 \pm 42.4$ & \\
\hline & $S-3$ & $65.3 \pm 19.7$ & $77.9 \pm 27.4$ & $84.7 \pm 32.6$ & \\
\hline \multirow{3}{*}{$\mathrm{SO}_{4}^{2-}$} & S-1 & $95.25 \pm 2.2$ & $106.5 \pm 8.9$ & $113.85 \pm 24.5$ & \multirow{3}{*}{400} \\
\hline & S-2 & $62.1 \pm 7.7$ & $70.17 \pm 12.7$ & $66.8 \pm 21.9$ & \\
\hline & S-3 & $60.05 \pm 3.7$ & $47 \pm 11.6$ & $55.35 \pm 18.7$ & \\
\hline & S-1 & $22.58 \pm 1.4$ & $23.4 \pm 2.7$ & $24.46 \pm 2.9$ & \multirow{3}{*}{10} \\
\hline \multirow[t]{3}{*}{$\mathrm{NO}_{3}{ }^{-}-\mathrm{N}$} & S-2 & $12.66 \pm 1.4$ & $19.18 \pm 1.21$ & $20.16 \pm 4.25$ & \\
\hline & S-3 & $10.3 \pm 1.7$ & $15.56 \pm 1.0$ & $11.3 \pm 2.77$ & \\
\hline & S-1 & $6.63 \pm 1.0$ & $6.98 \pm 0.44$ & $7.08 \pm 0.51$ & \multirow{4}{*}{$0.1 * *$} \\
\hline \multirow[t]{3}{*}{$\mathrm{PO}_{4}{ }^{3-}$} & S-2 & $3.305 \pm 0.35$ & $3.54 \pm 0.47$ & $3.81 \pm 0.32$ & \\
\hline & S-3 & $2.32 \pm 0.69$ & $2.24 \pm 0.6$ & $1.885 \pm 0.58$ & \\
\hline & S-1 & $88.8 \pm 5.8$ & $94.35 \pm 3.6$ & $95.44 \pm 3.03$ & \\
\hline \multirow[t]{3}{*}{$\mathrm{HCO}_{3}^{-}$} & S-2 & $52.64 \pm 5.8$ & $62.2 \pm 6.1$ & $59.9 \pm 8.3$ & \multirow[t]{2}{*}{-} \\
\hline & S-3 & $30.07 \pm 7.6$ & $36.0 \pm 12.0$ & $35.67 \pm 11.0$ & \\
\hline & S-1 & $30.38 \pm 0.89$ & $35.7 \pm 3.8$ & $35.5 \pm 2.2$ & \multirow{4}{*}{200} \\
\hline \multirow[t]{3}{*}{$\mathrm{Na}^{+}$} & S-2 & $22.69 \pm 4.1$ & $24.04 \pm 5.8$ & $24.9 \pm 4.1$ & \\
\hline & S-3 & $16.33 \pm 3.45$ & $21.5 \pm 7.5$ & $15.8 \pm 8.4$ & \\
\hline & S-1 & $67.78 \pm 15.1$ & $68.0 \pm 13.9$ & $68.9 \pm 12.6$ & \\
\hline \multirow[t]{3}{*}{$\mathrm{K}^{+}$} & S-2 & $41.8 \pm 11.4$ & $56.53 \pm 12.6$ & $41.7 \pm 12.7$ & \multirow[t]{2}{*}{12} \\
\hline & S-3 & $25.77 \pm 9.7$ & $47.15 \pm 12.1$ & $26.87 \pm 11.2$ & \\
\hline & S-1 & $23.39 \pm 3.17$ & $26.2 \pm 3.9$ & $29.3 \pm 6.7$ & \multirow{3}{*}{75} \\
\hline \multirow[t]{3}{*}{$\mathrm{Ca}^{2+}$} & S-2 & $17.16 \pm 5.5$ & $18.8 \pm 5.1$ & $18.04 \pm 5.2$ & \\
\hline & S-3 & $10.29 \pm 3.3$ & $12.2 \pm 4.3$ & $10.74 \pm 2.94$ & \\
\hline & S-1 & $7.35 \pm 0.6$ & $6.5 \pm 0.32$ & $7.81 \pm 1.5$ & \multirow{3}{*}{ - } \\
\hline \multirow[t]{2}{*}{$\mathrm{Mg}^{2+}$} & S-2 & $4.69 \pm 0.25$ & $4.01 \pm 0.58$ & $4.35 \pm 0.7$ & \\
\hline & S-3 & $3.27 \pm 0.34$ & $2.73 \pm 0.5$ & $3.61 \pm 0.27$ & \\
\hline
\end{tabular}

$* *$ [USEPA standard]

The maximum concentration of $\mathrm{Fe}, \mathrm{Cu}, \mathrm{Zn}, \mathrm{Mn}, \mathrm{Pb}, \mathrm{Cr}$, and $\mathrm{Cd}$ ions were $1.13,0.079,0.056,0.714,0.069,0.006$, and $0.016 \mathrm{mg} / \mathrm{L}$, respectively. Therefore, no heavy metal concentration exceeded the permissible limit in the study (Table 3).

Table 3. Heavy metals of surface water around the paper mill effluents discharge areas.

\begin{tabular}{|c|c|c|c|c|c|}
\hline \multirow{2}{*}{ Parameter } & \multirow{2}{*}{ Sample location } & \multicolumn{3}{|c|}{ Mean \pm SD } & \multirow{2}{*}{ BD SWS [24] } \\
\hline & & PM-1 & PM-2 & PM-3 & \\
\hline \multirow{3}{*}{$\mathrm{Fe}$} & S-1 & $1.03 \pm 0.1$ & $1.08 \pm 0.11$ & $1.13 \pm 0.29$ & \multirow{3}{*}{2} \\
\hline & S-2 & $0.93 \pm 0.31$ & $0.815 \pm 0.2$ & $0.812 \pm 0.23$ & \\
\hline & S-3 & $0.389 \pm 0.13$ & $0.453 \pm 0.17$ & $0.426 \pm 0.15$ & \\
\hline \multirow{3}{*}{$\mathrm{Cu}$} & S-1 & $0.07 \pm 0.00$ & $0.07 \pm 0.00$ & $0.079 \pm 0.02$ & \multirow{3}{*}{0.5} \\
\hline & S-2 & $0.045 \pm 0.00$ & $0.037 \pm 0.00$ & $0.06 \pm 0.02$ & \\
\hline & S-3 & $0.016 \pm 0.00$ & $0.027 \pm 0.00$ & $0.028 \pm 0.00$ & \\
\hline \multirow{3}{*}{$\mathrm{Zn}$} & S-1 & $0.052 \pm 0.00$ & $0.056 \pm 0.00$ & $0.054 \pm 0.00$ & \multirow{3}{*}{5} \\
\hline & S-2 & $0.026 \pm 0.00$ & $0.036 \pm 0.00$ & $0.035 \pm 0.00$ & \\
\hline & S-3 & $0.014 \pm 0.00$ & $0.026 \pm 0.00$ & $0.03 \pm 0.01$ & \\
\hline \multirow{3}{*}{$\mathrm{Mn}$} & S-1 & $0.714 \pm 0.10$ & $0.606 \pm 0.04$ & $0.556 \pm 0.07$ & \multirow{3}{*}{5} \\
\hline & S-2 & $0.349 \pm 0.08$ & $0.437 \pm 0.09$ & $0.455 \pm 0.1$ & \\
\hline & S-3 & $0.243 \pm 0.04$ & $0.230 \pm 0.09$ & $0.337 \pm 0.04$ & \\
\hline \multirow{3}{*}{$\mathrm{Pb}$} & S-1 & $0.069 \pm 0.00$ & $0.068 \pm 0.00$ & $0.067 \pm 0.00$ & \multirow{3}{*}{0.1} \\
\hline & S-2 & $0.056 \pm 0.00$ & $0.059 \pm 0.00$ & $0.048 \pm 0.00$ & \\
\hline & $S-3$ & $0.022 \pm 0.00$ & $0.035 \pm 0.00$ & $0.027 \pm 0.00$ & \\
\hline \multirow{3}{*}{$\mathrm{Cr}$} & S-1 & $0.005 \pm 0.00$ & $0.006 \pm 0.00$ & $0.006 \pm 0.00$ & \multirow{3}{*}{0.5} \\
\hline & S-2 & $0.003 \pm 0.00$ & $0.004 \pm 0.00$ & $0.004 \pm 0.00$ & \\
\hline & S-3 & $0.002 \pm 0.00$ & $0.003 \pm 0.00$ & $0.003 \pm 0.00$ & \\
\hline \multirow{2}{*}{$\mathrm{Cd}$} & S-1 & $0.011 \pm 0.00$ & $0.013 \pm 0.00$ & $0.016 \pm 0.00$ & \multirow{2}{*}{0.05} \\
\hline & S-2 & $0.009 \pm 0.00$ & $0.009 \pm 0.00$ & $0.009 \pm 0.00$ & \\
\hline
\end{tabular}




\section{Al-Nahrain Journal of Science}

ANJS, Vol.24 (3), September, 2021, pp. 63-72

\subsection{Physicochemical characterization of groundwater}

The physicochemical characteristics of groundwater around the paper mill effluents discharge areas are presented in Table 4. The average temperature of groundwater around PM-1, PM-2, and PM-3 were 25.5, 26.3, and $25.8{ }^{\circ} \mathrm{C}$, respectively within the Bangladesh drinking water standard (BDS). The average $\mathrm{pH}$ of PM-1, PM-2, and PM-3 groundwater were 7.17, 7.1, and 7.1, respectively were within the Bangladesh drinking water standard (BDS). Whereas the maximum DO level was $4.2 \mathrm{mg} / \mathrm{L}$ which is below the required level. The maximum EC and turbidity were $658 \mu \mathrm{S} / \mathrm{cm}$ and $4.82 \mathrm{NTU}$, respectively. There is no drinking water standard of EC, while the turbidity was within the standard level. The maximum level of TSS, TDS, BOD, COD, and TOC were 8.4, 449, 2.3, 8.27, and 1.3 $\mathrm{mg} / \mathrm{L}$, respectively, of these BOD and COD exceeded the permissible limit. Phenolic compounds were not detected (ND) level. The $\mathrm{Cl}^{-}, \mathrm{SO}_{4}{ }^{2-}, \mathrm{NO}_{3}{ }^{-}-\mathrm{N}, \mathrm{PO}_{4}{ }^{3-} \mathrm{Na}^{+}, \mathrm{K}^{+}, \mathrm{Ca}^{2+}$, and $\mathrm{Mg}^{2+}$ ions concentrations were within the permissible limit. The average concentration of $\mathrm{Mn}$ ion around PM-1, PM-2, and PM-3 groundwater were 0.146, 0.193, and 0.205 $\mathrm{mg} / \mathrm{L}$, respectively exceeded the Bangladesh drinking water standard (BDS) (Table 4). Hence, the groundwater of the area must be treated before drink. Whereas the $\mathrm{Fe}, \mathrm{Cu}, \mathrm{Zn}$, $\mathrm{Pb}, \mathrm{Cr}$, and $\mathrm{Cd}$ ions concentrations were within the permissible limit of drinking water standard in the study areas.

Table 4. Physicochemical parameters of groundwater around the paper mill effluent discharge areas

\begin{tabular}{|c|c|c|c|c|}
\hline \multirow{2}{*}{ Parameters } & \multicolumn{3}{|c|}{ Mean \pm SD } & \multirow{2}{*}{ BDS [24] } \\
\hline & PM-1 & PM-2 & PM-3 & \\
\hline Temperature & $25.5 \pm 6.3$ & $26.3 \pm 6.8$ & $25.8 \pm 6.5$ & $20-30$ \\
\hline $\mathrm{pH}$ & $7.17 \pm 0.15$ & $7.1 \pm 0.21$ & $7.1 \pm 0.22$ & $6.5-8.5$ \\
\hline DO & $4.2 \pm 0.06$ & $4.1 \pm 0.05$ & $4.2 \pm 0.1$ & $>6$ \\
\hline $\mathrm{EC}$ & $635 \pm 10.7$ & $658 \pm 40$ & $623 \pm 17$ & $1500^{*}$ \\
\hline Turbidity & $4.82 \pm 0.07$ & $4.6 \pm 0.21$ & $4.3 \pm 0.1$ & 10 \\
\hline TSS & $8.4 \pm 0.08$ & $7.3 \pm 0.89$ & $7.02 \pm 0.15$ & 10 \\
\hline TDS & $449 \pm 15.1$ & $449 \pm 15$ & $466 \pm 28$ & 1000 \\
\hline $\mathrm{BOD}_{5}$ & $2.3 \pm 0.12$ & $2.28 \pm 0.17$ & $2.2 \pm 0.07$ & 0.2 \\
\hline COD & $8.1 \pm 0.1$ & $8.27 \pm 0.14$ & $8.2 \pm 0.13$ & 4 \\
\hline TOC & $1.1 \pm 0.1$ & $1.3 \pm 0.46$ & $1.15 \pm 0.05$ & $2 * *$ \\
\hline Phenols & ND & ND & ND & 0.002 \\
\hline $\mathrm{Cl}^{-}$ & $45.6 \pm 2.1$ & $47.8 \pm 3.5$ & $41.9 \pm 3.4$ & 600 \\
\hline $\mathrm{SO}_{4}{ }^{2-}$ & $27.8 \pm 0.88$ & $27.9 \pm 2.3$ & $26.9 \pm 0.82$ & 400 \\
\hline $\mathrm{NO}_{3}{ }^{-}-\mathrm{N}$ & $2.47 \pm 0.17$ & $3.2 \pm 0.64$ & $3.02 \pm 0.32$ & 10 \\
\hline $\mathrm{PO}_{4}^{3-}$ & $1.96 \pm 0.09$ & $2.44 \pm 0.53$ & $1.43 \pm 0.432$ & 6 \\
\hline $\mathrm{HCO}_{3}^{-}$ & $13.3 \pm 1.5$ & $16.4 \pm 1.72$ & $18.8 \pm 2.8$ & - \\
\hline $\mathrm{Na}^{+}$ & $26.7 \pm 2.2$ & $26.8 \pm 2.8$ & $30.9 \pm 1.71$ & 200 \\
\hline $\mathrm{K}^{+}$ & $2.7 \pm 0.6$ & $2.7 \pm 0.55$ & $3.15 \pm 0.55$ & 12 \\
\hline $\mathrm{Ca}^{2+}$ & $26.3 \pm 2.4$ & $69.3 \pm 2.6$ & $69.5 \pm 3.9$ & 75 \\
\hline $\mathrm{Mg}^{2+}$ & $6.9 \pm 0.23$ & $7.85 \pm 0.64$ & $8.28 \pm 0.27$ & $30-35$ \\
\hline $\mathrm{Fe}$ & $0.44 \pm 0.09$ & $0.323 \pm 0.03$ & $0.419 \pm 0.07$ & $0.3-1.0$ \\
\hline $\mathrm{Cu}$ & $0.014 \pm 0.00$ & $0.019 \pm 0.00$ & $0.017 \pm 0.00$ & 1 \\
\hline $\mathrm{Zn}$ & $0.040 \pm 0.00$ & $0.06 \pm 0.02$ & $0.031 \pm 0.00$ & 5 \\
\hline $\mathrm{Mn}$ & $0.146 \pm 0.00$ & $0.193 \pm 0.00$ & $0.205 \pm 0.00$ & 0.1 \\
\hline $\mathrm{Pb}$ & $0.011 \pm 0.00$ & $0.02 \pm 0.00$ & $0.009 \pm 0.00$ & 0.05 \\
\hline $\mathrm{Cr}$ & $0.016 \pm 0.00$ & $0.012 \pm 0.00$ & $0.013 \pm 0.00$ & 0.05 \\
\hline $\mathrm{Cd}$ & $0.002 \pm 0.00$ & $0.002 \pm 0.00$ & $0.002 \pm 0.00$ & 0.005 \\
\hline
\end{tabular}

*[WHO standard], **[USEPA standard].

\subsection{Water quality index}

The Canadian Council of Ministers of the Environment (CCME) water quality index (WQI) was applied to assess the water quality around the paper mills effluents discharge areas. The standard values of the parameters were used to calculate the WQI score. The calculated terms of the CCME WQI for the surface water are stated in Table 5. The WQI score of the surface water ranged from 60.26 to 73.31 . At most of the sampling sites the water quality rankings were marginal, which indicated the surface water quality of those areas were threatened and often departed from the natural, or desirable levels. Whereas, few sampling sites having fair ranking indicated the quality of the water was usually protected but occasionally threatened and sometimes departed from natural, or desirable levels [18]. 


\section{Al-Nahrain Journal of Science}

ANJS, Vol.24 (3), September, 2021, pp. 63-72

\begin{tabular}{lcccccccc}
\multicolumn{6}{l}{ Table 5. The calculated terms of the CCME WQI for the surface water body around the paper mill effluents discharge areas } \\
\hline $\begin{array}{l}\text { Paper } \\
\text { mill }\end{array}$ & $\begin{array}{c}\text { Sample } \\
\text { location }\end{array}$ & Scope, F1 & $\begin{array}{c}\text { Frequency, } \\
\text { F2 }\end{array}$ & $\begin{array}{c}\sum \\
\text { Excursion }\end{array}$ & NSE & $\begin{array}{c}\text { Amplitude, } \\
\text { F3 }\end{array}$ & WQI score & Ranking \\
\hline PM-1 & S-1 & 36.36 & 36.36 & 107.48 & 0.81 & 44.88 & 60.59 & Marginal \\
& S-2 & 36.36 & 35.61 & 58.03 & 0.44 & 30.54 & 65.73 & Fair \\
& S-3 & 36.36 & 23.48 & 25.56 & 0.19 & 16.22 & 73.31 & Fair \\
PM-2 & S-1 & 36.36 & 36.36 & 111.36 & 0.84 & 45.76 & 60.26 & Marginal \\
& S-2 & 36.36 & 36.36 & 90.94 & 0.69 & 40.79 & 62.1 & Marginal \\
& S-3 & 36.36 & 36.36 & 72.57 & 0.55 & 35.47 & 63.93 & Marginal \\
PM-3 & S-1 & 36.36 & 36.36 & 110.32 & 0.84 & 45.53 & 60.35 & Marginal \\
& S-2 & 36.36 & 36.36 & 72.38 & 0.55 & 35.42 & 63.95 & Marginal \\
& S-3 & 36.36 & 24.24 & 30.93 & 0.23 & 18.98 & 72.49 & Fair \\
\hline
\end{tabular}

Figure 2 illustrates the CCME WQI scores of the surface water body around the paper mill effluents discharge areas. The WQI scores of the surface water around the paper mills show an increasing trend from site $\mathrm{S}-1$ to $\mathrm{S}-2$ to site $\mathrm{S}-3$. This trend infers that the paper mill effluents deteriorated the surface water quality at the discharge point $(\mathrm{S}-1)$ and gradually the water quality is improving concerning distance. Dinu et al. 2020; Al-Janabi et al. 2015 reported similar observations [27,28].

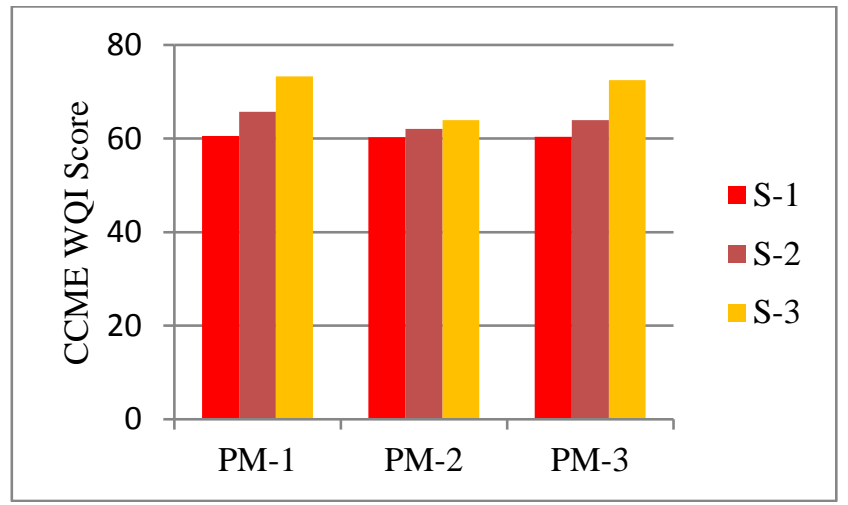

Figure 2. CCME WQI score of the surface water body around the paper mill effluents discharge areas.
The calculated terms of the CCME WQI for the groundwater are mentioned in Table 6. The WQI scores of the groundwater around the PM-1, PM-2, and PM-3 were 73.46, 73.12, and 73.48, respectively. At all the sampling sites the groundwater quality rankings were fair, which indicated the quality of the groundwater was usually protected but occasionally threatened and sometimes departed from natural, or desirable levels.

Table 6. The calculated terms of the CCME WQI for the groundwater around the paper mill effluents discharge areas.

\begin{tabular}{cccccccc}
\hline \multirow{2}{*}{ Paper mill } & \multirow{2}{*}{ Scope, F1 } & $\begin{array}{c}\text { Frequency, } \\
\text { F2 }\end{array}$ & E Excursion & NSE & $\begin{array}{c}\text { Amplitude, } \\
\text { F3 }\end{array}$ & WQI score & Ranking \\
\hline PM-1 & 19.05 & 19.05 & 74.8 & 0.59 & 37.25 & 73.46 & Fair \\
PM-2 & 19.05 & 19.05 & 77.12 & 0.61 & 37.97 & 73.12 & Fair \\
PM-3 & 19.05 & 19.05 & 74.67 & 0.59 & 37.21 & 73.48 & Fair \\
\hline
\end{tabular}

\subsection{Impacts of paper mill effluents}

The study found that the DO level was very low in the surface water around the paper mills area, which causes acute stress and death of aquatic organisms. High EC values indicate the presence of various ions dissolved in the water. The turbidity of the surface water was very high, which can harm aquatic life by reducing food supply, degrading spawning beds. It also affects the gill function of fishes [29]. The effluents containing a high level of TSS varied from 168.6 to $564.5 \mathrm{mg} / \mathrm{L}$, import a high level of BOD and COD. Moreover, TSS may change soil fertility, soil porosity, texture, and water holding capacity [30].
A high level of BOD and COD in the effluents indicated the presence of higher amounts inorganic and organic pollutants at a high level. Besides, High TOC values ranged from 48 to $105 \mathrm{mg} / \mathrm{L}$ in the effluents and surface water indicated the presence of organic pollutants at a high level. Moreover, phenolic compounds varied from 1.145 to 2.302 mg/L. Rigol et al., 2002; Terasaki et al., 2008; Criado et al. 2004; Vallejo et al. 2015 reported the presence of chlorophenols, amines, carboxylic acids, ethers, lignins, dioxin derivatives, furan derivatives and others toxic organic pollutants in paper mill effluents [13,31]. Toxic pollutants and heavy metals present in paper mill effluents cause biomagnification in the aquatic environment and 


\section{Al-Nahrain Journal of Science}

ANJS, Vol.24 (3), September, 2021, pp. 63-72

enter the human food chain through regular diets (Figure 3). Bioaccumulation of toxic components causes hepatotoxic, carcinogenic, cytotoxic, genotoxic effects, etc. [13]. In addition, adverse effects of the effluents on the aquatic ecosystem were reported by several researchers [32,33]. Paper mill effluents induce bacterial growth also [14,34]. Fang et al., 2012 reported that Ctylphenol, estrone, estradiol, nonylphenol, bisphenol A, triclosan, polychlorinated biphenyls (PCBs), polycyclic aromatic hydrocarbons (PAHs), polychlorinated dibenzo-p-dioxins (PCDD), and dibenzofurans (PCDF) presented in paper mill effluents may cause growth inhibition and death of fish [35]. Besides, reproductive toxicity, skin diseases, and other chronic toxicity to fish caused by several organic pollutants released from paper mill effluents were reported by Terasaki et al., 2012; Merilainen and Oikari, 2008 [36,37]. The positive impact of treated paper mill effluents irrigation on maize, barley, wheat sunflower, groundnut, and soybean production were reported in India [38,39]. But nail and skin problems of farmers, pathogenic infection, and bioaccumulation of toxic components may occur from wastewater usage for irrigation purposes [40,41]. Moreover, cytotoxic, genotoxic, mutagenic effects on Allium cepa, seed germination, and plant growth inhibition of toxic pollutants presented in paper mill effluents were reported $[13,25]$. Considering risk factors wastewater usage in irrigation purposes should not be encouraged in developing countries.

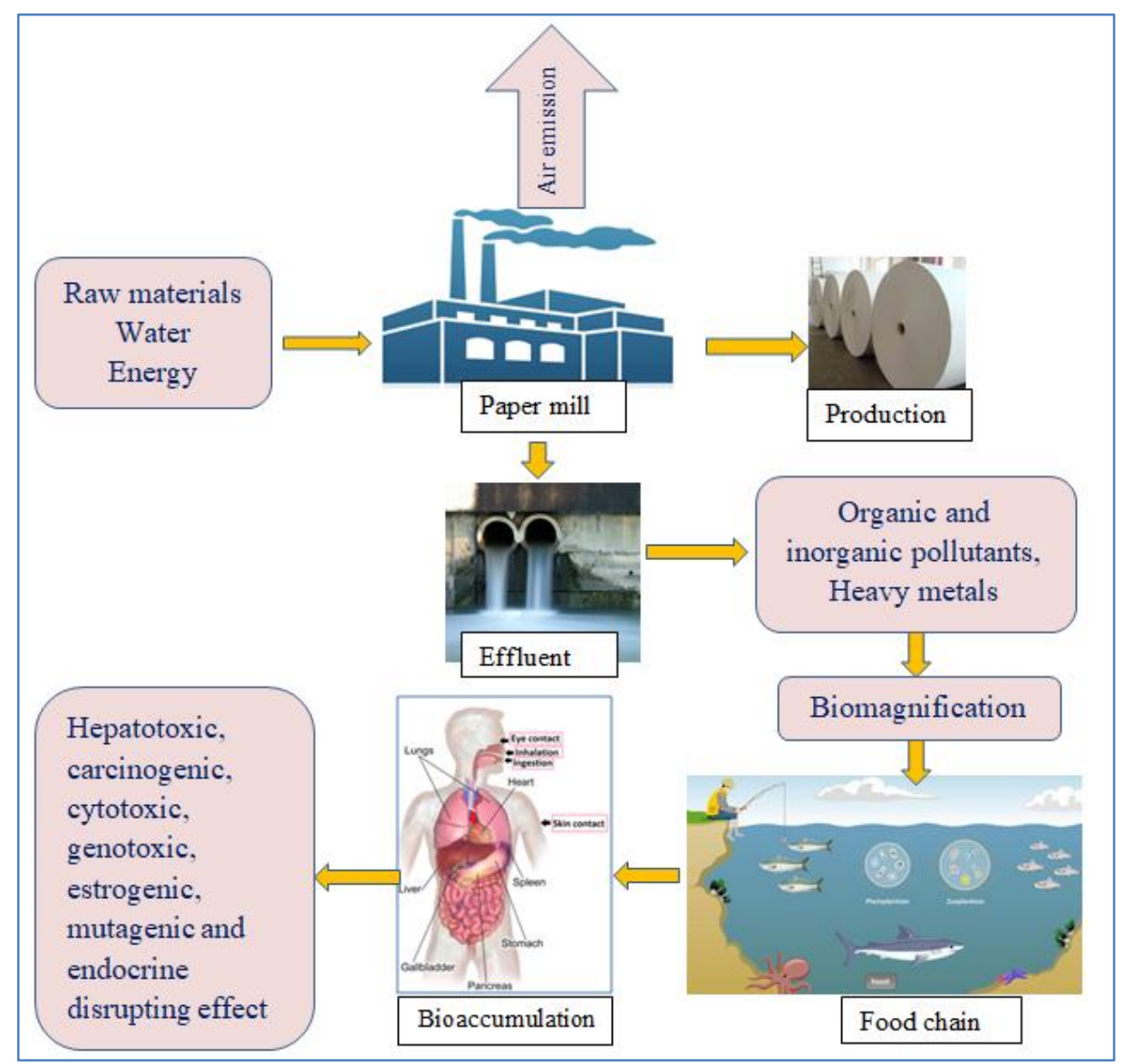

Figure 3. Bioaccumulation of toxic pollutants from paper mill effluents.

\section{Conclusion}

The study showed that the concentration of EC, TSS, BOD 5 , $\mathrm{COD}$, phenols, $\mathrm{NO}_{3}{ }^{-}-\mathrm{N}$, and $\mathrm{K}^{+}$ion in the surface water body around three (3) paper mills area exceeded the permissible limits of surface water standard. Moreover, the DO levels were found 1.63 to $3.5 \mathrm{mg} / \mathrm{L}$, which was below the standard level. The TOC values ranged from 48 to 105 $\mathrm{mg} / \mathrm{L}$ but, it has no surface water standard in Bangladesh. However, heavy metal concentrations were within the prescribed levels in the surface water body. The value of $\mathrm{COD}, \mathrm{BOD}_{5}$, and $\mathrm{Mn}$ in groundwater around the paper mills area exceeded the permissible limits of drinking water standards and suggested to treat before drinking. Besides, the DO levels showed below the standard level. The CCME WQI illustrated that the surface water quality at most sampling sites was within the 'marginal' category indicated that the water quality was threatening conditions. Besides, the groundwater quality was found within fair category, indicating that the water was safe at the moment. But it will become harmful for human consumption in the long run. The toxic pollutants of paper mill effluents entered into the aquatic environment eventually accumulated on the food chain, and the consequences are in bioaccumulation in the human body caused hepatotoxic, carcinogenic, cytotoxic, 


\section{Al-Nahrain Journal of Science}

ANJS, Vol.24 (3), September, 2021, pp. 63-72

genotoxic effects. Hence, regular monitoring of concerned authorities should be surveillance regularly, and the untreated effluents must be treated before discharging into the surface water bodies.

Data Availability: Derived data supporting the findings of this study are available from the corresponding author on request.

Conflict of Interest: The authors declare no conflict of interest.

Ethical statement: This article does not contain any experiment with any animal or human performed by any of the authors. The manuscript in part or in full has not been submitted or published anywhere and will not be submitted elsewhere until the editorial process is completed.

Funding statement: The study has not been received any funds from any organization.

\section{Reference}

[1] EU; "Best Available Techniques (BAT) Reference Document for the Production of Pulp". Paper and Board, European Union, Luxembourg, 2015.

[2] FAO; "Yearbook of Forest Products (2018)". Food and Agricultural Organization of the United Nations, Rome, 2020.

[3] Shakil M. S. Z. and Mostafa M. G. "Paper Industries Concern Water Pollution: A Review". International Journal of Innovative Research and Review, 9, 19-31, 2021.

[4] Rahman M. M.; and Kabir K. B; "Wastewater treatment options for paper mills using recycled paper/imported pulps as raw materials: Bangladesh perspective". Chemical Engineering Research Bulletin, 14(1), 65-68, 2010.

[5] Saha M. K. and Mostafa M. G.; "Impacts of Brick Kilns on Surface Water around Kiln Areas". Intl. J. of Multidisciplinary Sciences and Advanced Technology, 3(3), 1-18, 2021

[6] Waghmare S. M; Bhole A. G. and Dhabadgaonkar S. M.; "Evaluation of wastewater treatment plant of pulp and paper mill". IAWPC. The. Annul, 13, 51-54, 1986.

[7] Shakil M. S. Z.; Hasan M. R. and Mostafa M. G.; "Groundwater Exploitation and Its Environmental Consequences in Bangladesh: A Review". BAUET Journal, 2, 11-16, 2020.

[8] Yadav S. and Chandra R.; "Detection and assessment of the phytotoxicity of residual organic pollutants in sediment contaminated with pulp and paper mill effluent". Environmental monitoring and assessment, 190(10), 1-15, 2018.

[9] Islam M. R. and Mostafa M. G.; "Textile Dyeing Effluents and Environment Concerns-A Review". J. Environ. Sci. \& Natural Resources, 11(1\&2),131-144, 2018.

[10] Rahim M. A. and Mostafa M. G.; "Impact of sugar mills effluent on environment around mills area". AIMS Environmental Science, 8(1), 86-99, 2021.
[11] Helal Uddin S. M.; Mostafa M. G. and Haque A. B. M. H.; "Evaluation of groundwater quality and its suitability for drinking purpose in Rajshahi City, Bangladesh". Water Science and Technology: Water Supply, 11(5), 545-559, 2011. (IWA publication, London).

[12] Islam M. R. and Mostafa M. G.; "Characterization of textile dyeing effluent and its treatment using polyaluminum chloride". Applied Water Science, 10(5), 1-10, 2020.

[13] Singh A. K. and Chandra R.; "Pollutants released from the pulp paper industry: Aquatic toxicity and their health hazards". Aquatic toxicology, 211, 202-216. 2019.

[14] Devi N. L; Yadav I. C; Shihua Q. I; Singh S. and Belagali S. L.; "Physicochemical characteristics of paper industry effluents-a case study of South India Paper Mill (SIPM)". Environmental monitoring and assessment, 177(1), 23-33, 2011.

[15] Chowdhury M.; Mostafa M.G.; Biswas T. K. and Saha A. K.; "Treatment of leather industrial effluents by filtration and coagulation processes". Journal of Water Resource and Industry, 3, 11-22, 2013.

[16] Giri J.; Srivastava A.; Pachauri S. P. and Srivastava P. C.; "Effluents from paper and pulp industries and their impact on soil properties and chemical composition of plants in Uttarakhand, India". J Environ Waste Manag, 1, 26-32, 2014.

[17] Islam M. S. and Mostafa M. G.; "Groundwater Quality and Risk Assessment of Heavy Metal Pollution in Middle-West Part of Bangladesh". J. of Earth and Env. Sci. Res., 3(2), 1-5, 2021.

[18] CCME; "Canadian water quality guidelines for the protection of aquatic life: Canadian Water Quality Index 1.0 Technical Report. In Canadian environmental quality guidelines. 1999". Winnipeg. 2001.

[19] APHA; "Standard methods for the examination of the water and waste water". $21^{\text {st }}$ edn. APHA (American Public Health Association), AWWA, WPCF, Washington, DC 1134, 2005.

[20] Mostafa, M. G.; Helal Uddin S. M. and Haque A. B. M.; "Assessment of Hydro-geochemistry and Groundwater Quality of Rajshahi City in Bangladesh". Applied Water Science (Springer Pub), 7(8), 46634671, 2017.

[21] Saha M. K.; Sarkar R. R.; Ahmed S. J.; Sheikh A. H. and Mostafa M. G.; "Impacts of brick kiln emission on agricultural soil around brick kiln areas". Nepal Journal of Environmental Science, 9(1), 1-10, 2021.

[22] Mishra S.; Mohanty M.; Pradhan C.; Patra H. K.; Das R. and Sahoo S.; "Physico-chemical assessment of paper mill effluent and its heavy metal remediation using aquatic macrophytes-a case study at JK Paper mill, Rayagada, India". Environmental monitoring and assessment, 185(5), 4347-4359, 2013. 


\section{Al-Nahrain Journal of Science}

ANJS, Vol.24 (3), September, 2021, pp. 63-72

[23] Toczyłowska-Mamińska R.; "Limits and perspectives of pulp and paper industry wastewater treatment-A review". Renewable and Sustainable Energy Reviews, 78, 764-772, 2017.

[24] ECR; "Environmental Conservation Rules". Department of Environment. Ministry of Environment and Forest. People's Republic of Bangladesh. 1997.

[25] Chandra R.; Sharma P.; Yadav S. and Tripathi S.; "Biodegradation of Endocrine-Disrupting Chemicals and Residual Organic Pollutants of Pulp and Paper Mill Effluent by Biostimulation". Front. Microbiol., 9, 960, 2018.

[26] Kumar A.; Singhal V.; Joshi B. D. and Rai J. P. N.; "Impact of pulp and paper mill effluent on lysimetric soil and vegetation used for land treatment". Journal of Science and Industrial Research, 62, 883-891, 2003.

[27] Dinu C.; Scutariu R. E.; Vasile G.; Tenea A. G.; Petre J. and Cruceru L.; "Evaluation of wastewater quality using water quality index". Romanian Journal of Ecology \& Environmental Chemistry. 2 (2), 99-108, 2020.

[28] Al-Janabi Z. Z.; Al-Obaidy A. H. M. J. and Al-Kubaisi A. R.; "Applied of CCME Water Quality Index for Protection of Aquatic Life in the Tigris River within Baghdad city". Al-Nahrain Journal of Science, 18(2), 99-107, 2015.

[29] MPCA; "Turbidity: Description, Impact on Water Quality, Sources, Measures - A General Overview". Minnesota Pollution Control Agency, US. 2008.

[30] Chowdhury M.; Mostafa M. G.; Biswas T. K.; Mandal A.; Saha A. K.; "Characterization of the effluents from leather processing industries". Environmental Processes, 2(1), 173-187, 2015.

[31] Terasaki M.; Fukazawa H.; Tani Y. and Makino M.; "Organic pollutants in paper-recycling process water discharge areas: First detection and emission in aquatic environment". Environmental Pollution, 151(1), 53-59, 2008.

[32] Singh P.; Srivastava N.; Singh P.; Geetha S.; Usharani N.; Jagadish R. S.; Upadhyay A.; "Effect of Toxic Pollutants from Pulp \& Paper Mill on Water and Soil Quality and its Remediation". International Journal of Lakes and Rivers, 12(1), 1-20, 2019.

[33] Singh A.; Agarwal S. B.; Rai J. P. N. and Singh P.; "Assessment of the pulp and paper mill effluent on growth yield and nutrient quality of wheat (Triticum aestivum L.)". Journal of Environmental biology, 23 (3), 283-288, 2002.

[34] Beauchamp C. J.; Simao-Beaunoir A. M.; Beaulieu C. and Chalifour F. P.; "Confirmation of E. coli among other thermotolerant coliform bacteria in paper mill effluents, wood chips screening rejects and paper sludges". Water research, 40(12), 2452-2462, 2006.

[35] Fang Y. X.; Ying G. G.; Zhang L. J.; Zhao J. L.; Su H. C.; Yang B. and Liu S.; "Use of TIE techniques to characterize industrial effluents in the Pearl River
Delta region". Ecotoxicology and environmental safety, 76, 143-152, 2012.

[36] Terasaki M.; Jozuka K. and Makino M.; "Identification and accumulation of aromatic sensitizers in fish from paper recycling in Japan". Environmental toxicology and chemistry, 31(6), 12021208, 2012.

[37] Meriläinen P. and Oikari A.; "Uptake of organic xenobiotics by benthic invertebrates from sediment contaminated by the pulp and paper industry". Water research, 42(6-7), 1715-1725, 2008.

[38] Udayasoorian C.; Devagi P. and Ramaswami P. P.; "Case study on the utilization of paper and pulp mill effluent irrigation for field crop". In: Proceedings of Workshop on 'Bioremediation of Polluted Habitat's, 71-73, 1999.

[39] Juwarkar A. S. and Subramayam P. V. R.; "Impact of pulp and paper mill waste water on crop and soil". Water Sci. Tech., 19, 693-700, 1986.

[40] Van der Hoek W.; Ul Hassan M.; Ensink J. H. J.; Feenstra S.; Rashid-Sally L.; Munir S.; Aslam M.R.; Ali N.; Hussain R. and Matsuno Y.; "Urban wastewater: a valuable resource for agriculture". In: Research Report 63, IWMI, Sri Lanka, 2002.

[41] Trang D.T.; Van Der Hoek W.; Tuan N. D.; Cam P. D.; Viet V. H.; Luu D. D.; Konradsen F. and Dalsgaard A.; "Skin disease among farmers using wastewater in rice cultivation in Nam Dinh, Vietnam". Trop. Med. Int. Health 12 (s2), 51-58, 2007. 